\title{
Statin use is associated with carotid plaque composition: The Rotterdam Study
}

\author{
Blerim Mujaj a , Daniel Bos ${ }^{\text {a,b,e }}$, Mariana Selwaness ${ }^{\text {b }}$, Maarten J.G. Leening a,c,e ${ }^{\text {, }}$ Maryam Kavousi ${ }^{\text {a }}$, \\ Jolanda J. Wentzel ${ }^{\mathrm{d}}$, Aad van der Lugt ${ }^{\mathrm{b}}$, Albert Hofman ${ }^{\mathrm{e}}$, Bruno H. Stricker ${ }^{\mathrm{a}, *}$, \\ Meike W. Vernooij ${ }^{\mathrm{a}, \mathrm{b}}$, Oscar H. Franco ${ }^{\mathrm{a}}$
}

a Department of Epidemiology, Erasmus MC, Rotterdam, The Netherlands

${ }^{\mathrm{b}}$ Department of Radiology and Nuclear Medicine, Erasmus MC, Rotterdam, The Netherlands

c Department of Cardiology, Erasmus MC, Rotterdam, The Netherlands

d Department of Biomechanics, Erasmus MC, Rotterdam. The Netherlands

e Department of Clinical Epidemiology, Harvard TH Chan School of Public Health, Boston, USA

\section{A R T I C L E I N F O}

Article history:

Received 22 June 2017

Received in revised form 1 February 2018

Accepted 26 February 2018

Available online $\mathrm{xxxx}$

\section{Keywords:}

Carotid artery

Statin

Plaque composition

MRI

Atherosclerosis

Epidemiology

\begin{abstract}
A B S T R A C T
Background: Statins represent a key treatment for cardiovascular disease. Nevertheless, the direct effects of statin treatment on the composition of atherosclerotic plaques remain elusive.

Objectives: We aimed to investigate the association of statin treatment with the presence of different plaque components located in the carotid arteries within a population-based setting.

Methods: From the population-based Rotterdam Study, 1740 participants with carotid atherosclerosis (mean age 72.9 years, $46 \%$ women) underwent MRI of the carotid arteries to determine the presence of calcification, lipid core, and intraplaque hemorrhage. Information for the duration and dosage of statin use was obtained from pharmacy records for all participants. We used logistic regression models to study the association of statin use with the presence of plaque components.

Results: Statin treatment was associated with a higher presence of calcification (OR: 1.73 [95\% CI: 1.22-2.44]). Longer duration of use strengthened this association (OR: 1.82 [95\% CI: 1.00-3.33] for 10 to 48 months, and OR 1.74 [95\% CI: $1.09-2.77$ ] for $>48$ months, compared to OR: 1.65 [95\% CI: $0.94-2.89$ ] for $\leq 10$ months). Current statin treatment was also associated with a lower presence of lipid core (OR: 0.66 [95\% CI: 0.42-1.04]), but only when using statins for 10 months or less. Any dosage of statins was associated with a higher presence of calcification, whilst only high dosages (DDD $>1.33$ ) were associated with a lower presence of lipid core. Conclusions: Active, high-dosage statin use seems to beneficially influence the composition of carotid atherosclerosis by shifting the composition from vulnerable plaque with a lipid core to more stable calcified plaque.
\end{abstract}

@ 2017 Published by Elsevier B.V.

\section{Introduction}

Atherosclerosis in the carotid artery is the most important cause of stroke [1-3]. Within the complex etiological framework of atherosclerosis, a key role is played by serum low-density lipoprotein (LDL) cholesterol, specifically in the initiation and progression of the disease [2]. Following this, lowering the concentration of LDL cholesterol using statins has become a cornerstone for primary prevention of stroke and cardiovascular events overall $[4,5]$.

Several trials have demonstrated a direct effect of statins on the formation of coronary artery disease and a lower risk of coronary events

* Corresponding author at: Department of Epidemiology, Rm Na-2717, Erasmus MC - University Medical Center, PO Box 2040, 3000 CA Rotterdam, The Netherlands.

E-mail address: b.stricker@erasmusmc.nl (B.H. Stricker).
[6-8]. This direct effect of statins is thought to be due to the beneficial influence of statins on plaque stability by increasing the amount of calcium at the cost of vulnerable plaque components such as lipid core [9].

In contrast to the extensive research in the field of the coronary arteries, studies on the effects of statin treatment on atherosclerosis in the carotid arteries are far more limited, especially from a generalpopulation perspective. Yet, especially in light of the increased risk of stroke that carotid atherosclerosis harbors [10], it is paramount to also disentangle the effect of statin treatment on carotid artery atherosclerosis. Moreover, it is important to highlight that findings regarding the physiopathology of coronary artery disease may not be directly generalizable to the carotid arteries, given that correlation for atherosclerosis across vessel beds is only moderate [11,12]. Magnetic resonance imaging (MRI) allows detailed characterization of different plaque components, including lipid core, intraplaque hemorrhage (IPH), and calcification $[13,14]$. 
Against this background, we investigated the association of statin use with specific components of the carotid plaque in a large population-based sample of persons with subclinical atherosclerosis.

\section{Methods}

\subsection{Setting}

The current study is embedded within The Rotterdam Study, a prospective population-based cohort study, in participants of $\geq 45$ years living in Ommoord, a district of Rotterdam [15]. The Rotterdam Study has been approved by the medical ethics committee, according to the Population Screening Act: Rotterdam Study, executed by the Ministry of Health, Welfare and Sports of the Netherlands. All participants provided written informed consent.

\subsection{Study population}

Participants were selected on the basis of a carotid artery ultrasound examination (intima-media thickness $>2.5 \mathrm{~mm}$ in one or both carotid arteries) which is performed in all participants of the Rotterdam Study. Between the years 2007 and 2012, 2666 participants were invited to undergo an MRI examination of the carotid arteries. From the invited participants, 684 participants did not undergo MRI scanning due to claustrophobia $(n=57)$, physical limitations $(n=191)$, MRI contraindications $(n=115)$, refusal to participate $(n=272)$ and no show or lost to follow-up $(n=49)$, leaving 1982 participants. From these, we excluded another 242 participants due to poor image quality $(n=95)$, scan interruption due to claustrophobia $(n=106)$ and absence of plaque bilaterally ( $n=41$ ), leaving 1740 participants in the present analyses.

\subsection{Carotid scanning and analysis of plaque components}

MRI imaging was performed using a 1.5 Tesla scanner (GE Healthcare, Milwaukee, WI, USA) with a dedicated bilateral phase-array surface coil (Machnet, Eelde, the Netherlands). A standardized scanning protocol was used with a total scanning time of approximately $30 \mathrm{~min}$. The protocol included 4 sequences in axial plane: a proton density weighted (PDw)-fast spin echo (FSE)-black blood (BB) sequence (in-plane resolution $130 / 160 \times 130 / 128=0.8 \times 1 \mathrm{~cm})$; a PDw-FSE-BB with an increased in-plane resolution (in-plane resolution $130 / 224 \times 130 / 160=0.5 \times 0.8 \mathrm{~cm}$ ); a PDw-echo planar imaging (EPI) sequence (in-plane resolution 130/160 $\times 70 / 160=0.8 \times 0.4 \mathrm{~cm}$ ); a T2 weighted-EPI sequence (in-plane resolution $130 / 160 \times 70 / 160=0.8 \times 0.4 \mathrm{~cm}$ ) and two three-dimensional (3D) sequences: a 3D-T1 weighted (T1w)-gradient echo sequence (in-plane resolution $180 / 192 \times 180 / 180=0.9 \times 1 \mathrm{~cm}$ ), and a 3D phased-contrast magnetic resonance angiography (3D-PC-MRA) (in-plane resolution 180/256 $\times 180 / 128=$ $0.7 \times 1.4 \mathrm{~cm}$ ). Details of the scan protocol, scan reading procedure, and reproducibility are described in detail elsewhere [16]. We assessed plaque characteristics in all plaques with a maximum thickness of $\geq 2.5 \mathrm{~mm}$ on MRI. On the proton density weighted fast spin echo images, maximum carotid wall thickness was measured, and degree of luminal stenosis was calculated using the North American Symptomatic Carotid Endarterectomy Trial criteria [17]. The carotid images were evaluated for the presence of three different plaque components, calcification, lipid core, and IPH. Calcification was defined as the presence of a hypointense region in the plaque on all sequences [18-20]. IPH was defined as the presence of a hyperintense region in the atherosclerotic plaque on 3D-T1w-GRE $[21,22]$. Lipid core presence was defined as a hypointense region, not classified as IPH or calcification, in the plaque on PDw-FSE, PDw-EPI and T2w-EPI sequences, and with relative signal intensity drop on the T2w-EPI sequence $[18,19,23]$. All sequences were used in parallel to record the presence of plaque components. Subjects were recorded as positive for the presence of any plaque component if the component was identified in one or both carotid arteries. To test the intra-subject variability, 40 participants underwent a second MRI scan (average time between scans $15 \pm 9$ days) [16]. For an inter-observer reproducibility analysis, MRI examinations were selected randomly $(n=50)$ and read by a second independent observer with three years of experience. Inter-observer agreement and intra-scan agreement were calculated using Cohens' Kappa statistics. The intra-subject agreement was good for all measurements. The Kappa values were 0.95 (95\% CI 0.88-0.99) for the presence of intraplaque hemorrhage; 0.85 (95\% CI 0.74-0.96) for lipid core and 0.91 (95\% CI 0.82-0.99) for calcification [16]. Moreover, interobserver agreement was good for all measurements. The Kappa values were 0.86 (95\% CI 0.720.99 ) for intraplaque hemorrhage; 0.86 ( $95 \% \mathrm{Cl} 0.72-0.99$ ) for lipid core presence and 0.94 (95\% CI 0.86-0.99) for calcification [16].

\subsection{Assessment of statin treatment}

Information on statin treatment dispensing was obtained from fully computerized linked pharmacies in the study area. All prescriptions for statin therapy filed from January 1,1991 , until October 26, 2012, were available and included the product name of the drug, the anatomical therapeutic chemical code (ATC code), the amount dispensed, the prescribed dose regimen, and the date of dispensing. For every dispensing of statins, the duration of use (prescription episode) was calculated by dividing the number of dispensed tablets by the prescribed daily number. On the date of carotid MRI scanning, every participant was classified into one of the following mutually exclusive categories: 'current use' if the measurement occurred within a prescription episode; 'past use' if the participant had previously stopped using statins; or 'never use' if the participant had not used statins during the study period. Next, we created tertiles of the duration of cumulative exposure to statins among statin users. This resulted in the following categories: current use $\leq 10$ months; current use $10-48$ months; current use $>48$ months; past use $\leq 10$ months; past use $10-48$ months and past use $>48$ months since the end of the last prescription episode. To facilitate direct dose comparisons between drugs from the same therapeutic drug group, the daily defined dose (DDD) of statin therapy was expressed [24]. Finally, we created tertiles of discontinuation of statin use among past users as follows: $\leq 3$ months, $3-16$ months and $>16$ months.

\subsection{Other measurements in the Rotterdam Study}

Information on other relevant measurements was obtained by interview, physical examination, and blood sampling [15]. Smoking status was categorized into never, the past, and current smoking. Diabetes mellitus was defined as fasting blood glucose $>6.9 \mathrm{mmol} / \mathrm{L}$, nonfasting glucose $>11.0 \mathrm{mmol} / \mathrm{L}$, or use of glucoselowering medication. Systolic and diastolic blood pressure was measured using a random-zero sphygmomanometer on the right arm. Two measurements were performed and the average of the two was used in the analyses. Body mass index (BMI) was calculated based on weight in kilograms divided by height in meters squared. Serum total cholesterol and high-density lipoprotein (HDL) levels were measured using standard laboratory techniques. The use of antihypertensive medication and vitamin $\mathrm{K}$ antagonists (VKA) was obtained from pharmacy records [15].

\subsection{Statistical analysis}

We used a three-step statistical analysis approach to investigate the association between statin use and the presence of different plaque components. First, we used two logistic regression models to assess the association of statin use (never, former, current) with the presence of calcification, lipid core, and IPH in any of the two carotid arteries In the first model, we adjusted these analyses for age and sex. In the second model, we additionally adjusted for smoking, diabetes mellitus, systolic blood pressure, diastolic blood pressure, BMI, total cholesterol, HDL, use of antihypertensive medication, and use of vitamin $\mathrm{K}$ antagonists. Factors were selected based on previous literature and univariate analyses. Vitamin $\mathrm{K}$ antagonists were handled as a potential confounder given that these accelerate the deposition of calcification in the arterial wall through the competitive lowering of vitamin K receptor binding. Second, we investigated whether the duration of statin use was associated with any of the three plaque components, using the same regression models. For the duration of statin use, we compared the six categories as defined above (based on tertiles of use), vs. never use. Third, we investigated whether the DDD of statin treatment was associated with any of the three plaque components. Fourth, we created tertiles of DDD of statin treatment and compared the three tertile categories with never use. Finally, we investigated the association of discontinuation of statin use with plaque composition (based on tertiles of discontinuation).

Additionally, we performed sensitivity analyses to address confounding by indication, we re-analyzed all associations in participants without prevalent cardiovascular diseases (participants with a confirmed history of stroke, myocardial infarction, and coronary heart disease) (Table 1) [4,5]. Finally, we performed stratified analyses for age below and above 70 years of age and sex, to investigate whether associations differed by these

\section{Table 1}

Baseline characteristics of the study population $(n=1740)$.

\begin{tabular}{llll}
\hline & Never user & Current user & Past user \\
& $N=1014$ & $N=526$ & $N=200$ \\
\hline Age (years) & $72.05 \pm 9.9$ & $73.60 \pm 8.0$ & $75.44 \pm 6.8$ \\
Women (\%) & 48.8 & 38.2 & 52.0 \\
Current smoking (\%) & 30.8 & 33.5 & 30.0 \\
Diabetes mellitus (\%) & 8.2 & 24.0 & 21.0 \\
Systolic blood pressure (mm/Hg) & $144.6 \pm 20$ & $146.1 \pm 21$ & $148.4 \pm 20$ \\
Diastolic blood pressure (mm/Hg) & $80.7 \pm 10$ & $79.2 \pm 10$ & $80.1 \pm 12$ \\
BMI (kg/m ${ }^{2}$ ) & $27.0 \pm 3.6$ & $27.6 \pm 3.2$ & $27.9 \pm 3.7$ \\
Total cholesterol (mmol/L) & $5.83 \pm 0.8$ & $5.22 \pm 1.1$ & $5.65 \pm 1.1$ \\
HDL cholesterol (mmol/L) & $1.46 \pm 0.3$ & $1.29 \pm 0.3$ & $1.37 \pm 0.3$ \\
Antihypertensive medication use (\%) & 27.4 & 59.1 & 47.5 \\
Vitamin K antagonists use (\%) & 4.2 & 8.4 & 5.0 \\
History of stroke (\%) & 2.5 & 12.0 & 10.5 \\
History of myocardial infarction $(\%)$ & 1.9 & 21.5 & 20.0 \\
History of CHD (\%) & 1.4 & 26.8 & 22.0 \\
Degree of stenosis (\%) mean $\pm \mathrm{SD}$ & $16.1 \pm 17.5$ & $22.3 \pm 23.3$ & $20.4 \pm 21.4$ \\
Wall thickness, mm & $3.1 \pm 0.6$ & $3.3 \pm 0.7$ & $3.3 \pm 0.8$ \\
Presence of calcification & 78.7 & 88.4 & 84.5 \\
Presence of lipid core & 45.0 & 42.4 & 43.5 \\
Presence of IPH & 30.7 & 39.5 & 42.0 \\
\hline
\end{tabular}

Values are means with standard deviations for continuous variables and percentages for dichotomous or categorical variables.

Abbreviations: $\mathrm{BMI}=$ body mass index, $\mathrm{CHD}=$ coronary heart disease, $\mathrm{HDL}=$ High density lipoprotein. 
factors. All analyses were carried out using IBM SPSS Statistical package version 21 (Chicago, IL, USA).

\section{Results}

Table 1 presents the baseline characteristics of the study population. Among study subjects a total of $30.2 \%$ were on statin treatment at the time of MRI. In statin users, the median duration of exposure to statins was 48 months (interquartile range, 10-110 months) with varying DDD regimes. Whereas in the statin past users the median statin discontinuation was 16 months (interquartile range, 3-48 months).

\subsection{Statin use and plaque composition}

Current statin use was associated with the presence of calcification and lipid core (age, sex and carotid wall thickness adjusted odds ratios [OR]: 1.77 , and 0.78 , respectively). After additional adjustment for cardiovascular risk factors, only the association between current statin use and the presence of calcification remained statistically significant (OR: 1.73 [95\% CI: 1.22-2.44]) (Table 2). We found no association between current statin use and the presence of IPH. Moreover, we found no association of past statin use with any of the plaque characteristics.

\subsection{Duration of statin use and plaque composition}

Longer duration of statin use was associated with a higher presence of calcification (for statin use of 10 months OR: 1.65 [95\% CI: 0.94-2.89]), (for statin use of 10 to 48 months OR: 1.82 [95\% CI: 1.00-3.33]), and (for statin use of $>48$ months OR: 1.74 [95\% CI: 1.09-2.77]) (p-trend 0.01) (Table 3). We also found an association between the current statin use of $\leq 10$ months with a lower presence of lipid core (OR: 0.54 [95\% CI: 0.35-0.83]). After additional adjustment this association was not significant (OR: $0.66[95 \%$ CI: $0.42-1.04]$ ). We found no associations for any length of past use with any of the plaque components (Table 3 ).

\subsection{Dosage of statin use and plaque composition}

We found a dose-response relation between the DDD of statin use and a higher presence of calcification and a lower presence of lipid core (Fig. 1). The high statin dosage did not associate with a presence of IPH, even after adjustment for cardiovascular risk factors (Fig. 1).

\subsection{Discontinuation of statin use and plaque composition}

When we examined associations between the discontinuation of statin use in past users, we found a positive trend of $\leq 3$ months of statin discontinuation with a higher presence of calcification (OR: 1.48 [95\% $\mathrm{CI}: 0.60-3.61])$ and the positive trend of statin discontinuation of more than $>16$ months with a lipid core. Interestingly, we also found

Table 2

Association between statin treatment and carotid artery plaque composition.

\begin{tabular}{llll}
\hline Statin use & Calcification & Lipid core & IPH \\
& OR $(95 \% \mathrm{CI})$ & OR $(95 \% \mathrm{CI})$ & OR $(95 \% \mathrm{CI})$ \\
\hline Never use & Ref & Ref & Ref \\
Current use & $1.73(1.22-2.44)$ & $0.91(0.72-1.16)$ & $1.07(0.82-1.40)$ \\
Past use & $1.13(0.73-1.75)$ & $0.99(0.71-1.37)$ & $1.35(0.94-1.92)$ \\
Global $P$-value over & $<0.05$ & $>0.05$ & $<0.05$ \\
$\quad$ statin use categories & & & \\
\hline
\end{tabular}

All estimates were adjusted for age, sex, carotid wall thickness, smoking, diabetes mellitus, systolic blood pressure, diastolic blood pressure, body mass index, total cholesterol, high-density lipoprotein, blood pressure-lowering medication use and vitamin $\mathrm{K}$ antagonist use.

Abbreviations: $\mathrm{CI}=$ confidence interval, $\mathrm{IPH}=$ intra-plaque hemorrhage, $\mathrm{OR}=$ odds ratio.
Table 3

Association between duration of statin treatment and plaque composition.

\begin{tabular}{llll}
\hline Statin use & $\begin{array}{l}\text { Calcification } \\
\text { OR }(95 \% \mathrm{CI})\end{array}$ & $\begin{array}{l}\text { Lipid core } \\
\text { OR }(95 \% \mathrm{CI})\end{array}$ & $\begin{array}{l}\text { IPH } \\
\text { OR }(95 \% \mathrm{CI})\end{array}$ \\
\hline Never use & Ref & Ref & Ref \\
Current $\leq 10$ months & $1.65(0.94-2.89)$ & $0.66(0.42-1.04)$ & $1.25(0.76-2.04)$ \\
Current $10-48$ months & $1.82(1.00-3.33)$ & $0.95(0.64-1.40)$ & $0.81(0.52-1.27)$ \\
Current $>48$ months & $1.74(1.09-2.77)$ & $1.00(0.74-1.34)$ & $1.14(0.83-1.58)$ \\
Past $\leq 10$ months & $0.82(0.44-1.52)$ & $1.04(0.63-1.71)$ & $1.89(1.11-3.22)$ \\
Past $10-48$ months & $1.50(0.65-3.45)$ & $1.28(0.72-2.25)$ & $1.79(0.97-3.29)$ \\
Past $>48$ months & $1.37(0.65-2.90)$ & $0.76(0.45-1.29)$ & $0.71(0.39-1.30)$ \\
Global P-value over & $<0.05$ & $>0.05$ & $<0.05$ \\
$\quad$ statin use categories & & & \\
\hline
\end{tabular}

All estimates were adjusted for age, sex, carotid wall thickness, smoking, diabetes mellitus, systolic blood pressure, diastolic blood pressure, body mass index, total cholesterol, high-density lipoprotein, blood pressure-lowering medication use and vitamin $\mathrm{K}$ antagonist use.

Abbreviations: $\mathrm{CI}=$ confidence interval, $\mathrm{IPH}=$ intra-plaque hemorrhage, $\mathrm{OR}=$ odds ratio.

that discontinuation of longer than 3 months (i.e. 3-16 months) was associated with a higher presence of IPH (OR: 1.66 [95\% CI: 1.05-2.64]) (Supplementary Table 1).

\subsection{Sensitivity and stratified analyses}

After excluding participants with a history of CVD, the results did not substantially change (Supplementary Table 2). When we compared the association of statin use with the presence of calcification among persons younger than $\leq 70$ vs. those older than $>70$, we found that current use and past use were associated most prominently with the presence of calcification and IPH in persons younger than $\leq 70$ (Supplementary Table 3). We found no differences between men and women (Supplementary Table 4).

\section{Discussion}

In this large sample of middle-aged and elderly communitydwelling individuals, we observed that current use of a statin is related to a higher presence of calcification and a lower presence of lipid core in the carotid atherosclerotic plaque. A longer duration of statin use was primarily associated with the presence of calcification. Moreover, higher dosages of statins were related to a higher presence of calcification and a lower presence of lipid core. We found no influence of past statin use on carotid plaque components.

The current findings suggest that statins may play an important role in the remodeling of the atherosclerotic plaque composition and potentially change the natural course of atherosclerotic disease in the carotid artery. Thus far, evidence on the role of statins comes from several clinical studies investigating the effect of statins on cardiovascular disease [6-8], mainly targeting coronary arteries and the risk of coronary heart disease [25-27]. Primarily, these studies consistently demonstrated that plaques tend to regress (i.e. Diminish in size) under the influence of statins and statins play a role in risk reduction of atherosclerotic cardiovascular events [28-32]. However, evidence from trials with patients, targeting carotid artery, demonstrated that statin treatment markedly reduced intima-media thickness, plaque volume, lipid core and increased calcification [28,33-35]. Collectively, these studies examined the effect of statins and attributed the beneficial role of statins based on their ability to reduce cardiovascular events, but did not elucidate the direct effect of statins on plaque composition. Our study differed from these prior investigations, particularly as we investigated in detail the plaque components of atherosclerotic plaques. In this context, our findings indicate that statins inhibit progression of vulnerable components (i.e. lipid core in the atherosclerotic plaque), promotes calcification of the plaque and here with the transition from vulnerable to the stable plaque. Similar findings have been reported 


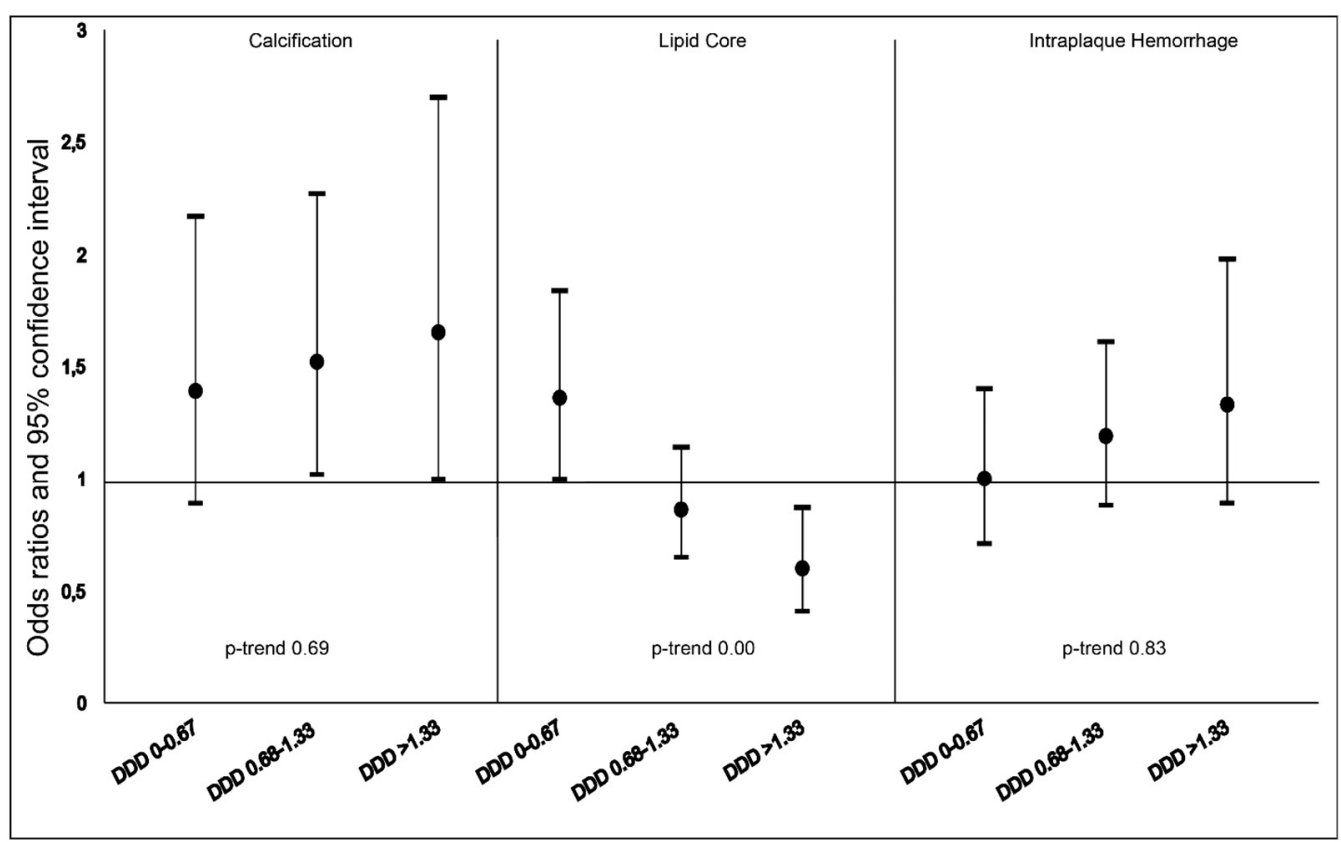

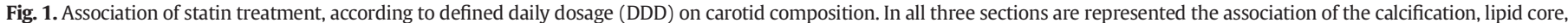

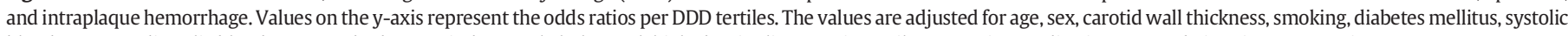
blood pressure, diastolic blood pressure, body mass index, total cholesterol, high-density lipoprotein, antihypertensive medication use, and vitamin $\mathrm{K}$ antagonist use.

by a Multicenter CONFIRM study, which investigated the effects of statins on coronary plaque composition [36]. In support of our findings a similar trend with calcification, lipid core, and intraplaque hemorrhage has been reported by the study, within the Rotterdam Study, when assessed the effect of statins on the incidence or the persistence of calcification, lipid core and IPH after 4 years of follow-up in longitudinal design, but owing to a small study sample limited the capacity to detect associations [37]. Although our study design was cross-sectional, we may hypothesize that statins remodel the atherosclerotic plaque composition, thereby, accelerate calcification of the plaque under statin treatment whilst at the same time, decrease lipid core. However, pathological observations suggest the central role of the vascular smooth muscle cells and macrophage apoptosis as driving mechanisms of calcification [38]. Aside from lipid regression within a plaque statin treatment may promote calcification in the plaque, herein plaque stabilization [25]. Similarly, recent PET studies related effects of statins with reduced vascular inflammation and vascular calcification $[39,40]$. Therefore, it is likely that the beneficial role of statins may be attributed to the capacity of statins to remodel the composition of the atherosclerotic plaque towards more calcification and less non-calcified components. Importantly, the amount of calcification as a measure of plaque-stability and a potential indicator of lower risk of subsequent clinical events requires further research [41].

Statin treatment produces observable changes in the plaque after short periods of use, nevertheless, it remains unclear to what extent of the duration of use statins can alter plaque morphology into a stable plaque phenotype. In the current study, we were able to consider the three different cutoffs for the duration of use and dosage. These findings suggest that statins are capable of promoting calcification in the short term of use, in low and high dosages, whereas the regression of lipid core can be achieved only through high dosages [42]. Alternatively, weighing the p-trends we may hypothesize that duration of use significantly relates to calcification component and dosage of use relates to lipid component. At this point, an important clinical finding has been reported from a middle-aged patient who presented with moderate ischemia and coronary computed tomography (CTA) revealed a large amount of atherosclerotic plaque in the proximal left circumflex coronary artery. Among the others, the patient has been put in statin high dosage treatment [43]. After 4 years the same patient returned back, the coronary CTA was performed, and it showed a marked reduction of plaque amount [43]. Similarly, our findings may suggest that a longer duration and high dosages of statin treatment should be considered to lower the risk of atherosclerotic CVD on primary prevention [44-46]. Furthermore, we highlight that duration and daily dosage of statin treatment are key factors to determine the direction of plaque remodeling process and plaque morphology.

In contrast to our findings with current statin use, we did not find evidence for beneficial effects of past statin use suggesting a narrower window of exposure to treatment in order to accrue these effects. This is underlined by our finding that discontinuation of 3 months or longer was associated with a higher presence of vulnerable components, namely lipid core, and intraplaque hemorrhage. This may thus indicate that cessation of statin treatment will eventually lead to the continuation of plaque development, which could be comparable to the development prior to statin use. These findings are in line with a recent study in which the discontinuation of statin use led to an increased risk of myocardial infarction and cardiovascular mortality [47]. Following this, future longitudinal studies that take into account the effect of statin use on clinical outcomes such as cardiovascular events and mortality, are warranted.

The major strength is that our study is the first population-based study, with a long duration of exposure to statins, to investigate the association of statin use with carotid plaque components in a relatively large study sample. All data were collected irrespective of the current study. Furthermore, we were able to characterize in detail each specific component of the carotid atherosclerotic plaque using MRI. Additionally, we were able to take into consideration the duration and dosing of statin therapy. Yet, some considerations with regard to our study should also be addressed. First, although we conducted a sensitivity analysis confounding by indication in participants with a history of CVD should be considered. Second, due to the observational nature of the study, our findings should be regarded as hypothesis-generating and nonconclusive. Third, we were not able to consider the quantitative measures of each component within the atherosclerotic plaque. The quantitative assessments may provide additional, unique information on plaque composition and that future endeavors will also involve 
quantitative plaque assessments. Fourth, the potential of the MRI or CT versus PET/CT on detection of microcalcification is weak [48], therefore the interpretation of our results on calcification should be in the context of the macrocalcification.

\section{Conclusions}

In summary, we found that current statin treatment and high dosages of statins seem to beneficially influence the composition of the atherosclerotic plaque in the carotids by lowering the presence of the vulnerable lipid core component and contributing to a higher presence of stable calcified plaques.

\section{Authors' contributions}

Study concept and design: B.M., D.B., O.H.F. Acquisition, analysis or interpretation of data: B.M., D.B., B.H.S., O.H.F. Drafting of the manuscript: B.M. Critical revision of the manuscript for important intellectual content: B.M., D.B.,M.S., M.J.G.L.,M.K., J.W., A.V.L.,A.H., B.H.S., M.W.V., O.H.F. Statistical analysis: B.M. Administrative, technical, or material support: B.M., D.B., O.H.F. All authors read and approved the final manuscript.

\section{Acknowledgments}

The dedication, commitment, and contribution of the inhabitants, general practitioners, and pharmacists of the Ommoord district to the Rotterdam Study are gratefully acknowledged.

\section{Sources of funding}

The Rotterdam Study is supported by the Erasmus MC and Erasmus University Rotterdam; the Netherlands Organisation for Scientific Research (NWO); the Netherlands Organisation for Health Research and Development (ZonMw); the Research Institute for Diseases in the Elderly (RIDE); the Netherlands Genomics Initiative (NGI); the Ministry of Education, Culture and Science, Netherlands, the Ministry of Health, Welfare and Sport; the European Commission (DG XII); and the Municipality of Rotterdam.

Mr. Mujaj is supported by Erasmus Mundus Western Balkans (ERAWEB), a project funded by the European Commission.

Maryam Kavousi is supported by The Netherlands Organisation for Scientific Research Grant VENI, 91616079.

None of the funders had any role in the design and conduct of the study; collection, management, analysis, and interpretation of the data; and preparation, review, or approval of the manuscript.

\section{Conflict of interest}

The authors report no potential conflicts of interest.

\section{Appendix A. Supplementary data}

Supplementary data to this article can be found online at https://doi. org/10.1016/j.ijcard.2018.02.111.

\section{References}

[1] D. Mozaffarian, E.J. Benjamin, A.S. Go, D.K. Arnett, M.J. Blaha, M. Cushman, et al., Executive summary: heart disease and stroke statistics-2016 update: a report from the American Heart Association, Circulation 133 (2016) 447-454

[2] L. Badimon, G. Vilahur, LDL-cholesterol versus HDL-cholesterol in the atherosclerotic plaque: inflammatory resolution versus thrombotic chaos, Ann. N. Y. Acad. Sci. 1254 (2012) 18-32.

[3] D. Bos, M.J. Leening, M. Kavousi, A. Hofman, O.H. Franco, A. van der Lugt, et al., Comparison of atherosclerotic calcification in major vessel beds on the risk of all-cause and cause-specific mortality: the Rotterdam Study, Circ. Cardiovasc. Imaging 8 (2015).
[4] A.L. Catapano, I. Graham, G. De Backer, O. Wiklund, M.J. Chapman, H. Drexel, et al., 2016 ESC/EAS guidelines for the management of dyslipidaemias, Eur. Heart J. 37 (2016) 2999-3058.

[5] N.J. Stone, J.G. Robinson, A.H. Lichtenstein, C.N. Bairey Merz, C.B. Blum, R.H. Eckel, et al. 2013 ACC/AHA guideline on the treatment of blood cholesterol to reduce atherosclerotic cardiovascular risk in adults: a report of the American College of Cardiology/American Heart Association Task Force on Practice Guidelines, J. Am. Coll. Cardiol. 63 (2014) 2889-2934.

[6] Randomised trial of cholesterol lowering in 4444 patients with coronary heart disease: the Scandinavian Simvastatin Survival Study (4S), Lancet 344 (1994) 1383-1389.

[7] M.A. Pfeffer, F.M. Sacks, L.A. Moye, L. Brown, J.L. Rouleau, L.H. Hartley, et al., Cholesterol and Recurrent Events: a secondary prevention trial for normolipidemic patients. CARE Investigators, Am. J. Cardiol. 76 (1995) 98C-106C.

[8] J. Shepherd, S.M. Cobbe, I. Ford, C.G. Isles, A.R. Lorimer, P.W. MacFarlane, et al., Prevention of coronary heart disease with pravastatin in men with hypercholesterolemia. West of Scotland Coronary Prevention Study Group, N. Engl. J. Med. 333 (1995) 1301-1307.

[9] C. Baigent, A. Keech, P.M. Kearney, L. Blackwell, G. Buck, C. Pollicino, et al., Efficacy and safety of cholesterol-lowering treatment: prospective meta-analysis of data from 90,056 participants in 14 randomised trials of statins, Lancet 366 (2005) 1267-1278.

[10] A. Gupta, H. Baradaran, A.D. Schweitzer, H. Kamel, A. Pandya, D. Delgado, et al., Carotid plaque MRI and stroke risk: a systematic review and meta-analysis, Stroke 44 (2013) 3071-3077.

[11] D. Bos, M.A. Ikram, S.E. Elias-Smale, G.P. Krestin, A. Hofman, J.C. Witteman, et al. Calcification in major vessel beds relates to vascular brain disease, Arterioscler. Thromb. Vasc. Biol. 31 (2011) 2331-2337.

[12] M.A. Allison, M.H. Criqui, C.M. Wright, Patterns and risk factors for systemic calcified atherosclerosis, Arterioscler. Thromb. Vasc. Biol. 24 (2004) 331-336.

[13] H. El Aidi, V. Mani, K.B. Weinshelbaum, S.H. Aguiar, H. Taniguchi, J.E. Postley, et al., Cross-sectional, prospective study of MRI reproducibility in the assessment of plaque burden of the carotid arteries and aorta, Nat. Clin. Pract. Cardiovasc. Med. 6 (2009) 219-228

[14] C. Yuan, K.W. Beach, L.H. Smith Jr., T.S. Hatsukami, Measurement of atherosclerotic carotid plaque size in vivo using high resolution magnetic resonance imaging, Circulation 98 (1998) 2666-2671.

[15] A. Hofman, G.G. Brusselle, S. Darwish Murad, C.M. van Duijn, O.H. Franco, A. Goedegebure, et al., The Rotterdam Study: 2016 objectives and design update, Eur. J. Epidemiol. 30 (2015) 661-708.

[16] Q.J. van den Bouwhuijsen, M.W. Vernooij, A. Hofman, G.P. Krestin, A. van der Lugt, J.C. Witteman, Determinants of magnetic resonance imaging detected carotid plaque components: the Rotterdam Study, Eur. Heart J. 33 (2012) 221-229.

[17] I.N. Staikov, M. Arnold, H.P. Mattle, L. Remonda, M. Sturzenegger, R.W. Baumgartner, et al., Comparison of the ECST, CC, and NASCET grading methods and ultrasound for assessing carotid stenosis. European Carotid Surgery Trial. North American Symptomatic Carotid Endarterectomy Trial, J. Neurol. 247 (2000) 681-686.

[18] V.C. Cappendijk, K.B. Cleutjens, A.G. Kessels, S. Heeneman, G.W. Schurink, R.J. Welten, et al., Assessment of human atherosclerotic carotid plaque components with multisequence MR imaging: initial experience, Radiology 234 (2005) 487-492.

[19] T. Saam, M.S. Ferguson, V.L. Yarnykh, N. Takaya, D. Xu, N.L. Polissar, et al., Quantitative evaluation of carotid plaque composition by in vivo MRI, Arterioscler. Thromb. Vasc. Biol. 25 (2005) 234-239.

[20] B. Mujaj, A.M. Lorza, A. van Engelen, M. de Bruijne, O.H. Franco, A. van der Lugt, et al., Comparison of CT and CMR for detection and quantification of carotid artery calcification: the Rotterdam Study, J. Cardiovasc. Magn. Reson. 19 (2017) 28.

[21] R. Bitar, A.R. Moody, G. Leung, S. Symons, S. Crisp, J. Butany, et al., In vivo 3D highspatial-resolution MR imaging of intraplaque hemorrhage, Radiology 249 (2008) 259-267.

[22] A.R. Moody, Magnetic resonance direct thrombus imaging, J. Thromb. Haemost. 1 (2003) 1403-1409.

[23] C. Yuan, L.M. Mitsumori, M.S. Ferguson, N.L. Polissar, D. Echelard, G. Ortiz, et al., In vivo accuracy of multispectral magnetic resonance imaging for identifying lipid-rich necrotic cores and intraplaque hemorrhage in advanced human carotid plaques, Circulation 104 (2001) 2051-2056.

[24] C.E. de Keyser, F.V. de Lima, F.H. de Jong, A. Hofman, Y.B. de Rijke, A.G. Uitterlinden, et al., Use of statins is associated with lower serum total and non-sex hormonebinding globulin-bound testosterone levels in male participants of the Rotterdam Study, Eur. J. Endocrinol. 173 (2015) 155-165.

[25] R. Puri, S.J. Nicholls, M. Shao, Y. Kataoka, K. Uno, S.R. Kapadia, et al., Impact of statins on serial coronary calcification during atheroma progression and regression, J. Am. Coll. Cardiol. 65 (2015) 1273-1282.

[26] S. Auscher, L. Heinsen, K. Nieman, K.H. Vinther, B. Logstrup, J.E. Moller, et al., Effects of intensive lipid-lowering therapy on coronary plaques composition in patients with acute myocardial infarction: assessment with serial coronary CT angiography, Atherosclerosis 241 (2015) 579-587.

[27] M. Banach, C. Serban, A. Sahebkar, D.P. Mikhailidis, S. Ursoniu, K.K. Ray, et al., Impact of statin therapy on coronary plaque composition: a systematic review and meta-analysis of virtual histology intravascular ultrasound studies, BMC Med. 13 (2015) 229.

[28] R.Q. Migrino, M. Bowers, L. Harmann, R. Prost, LaDisa JF Jr., Carotid plaque regression following 6-month statin therapy assessed by 3T cardiovascular magnetic resonance: comparison with ultrasound intima media thickness, J. Cardiovasc. Magn. Reson. 13 (2011) 37.

[29] R. Corti, Z.A. Fayad, V. Fuster, S.G. Worthley, G. Helft, J. Chesebro, et al., Effects of lipid-lowering by simvastatin on human atherosclerotic lesions: a longitudinal 
study by high-resolution, noninvasive magnetic resonance imaging, Circulation 104 (2001) 249-252.

[30] R. Corti, V. Fuster, Z.A. Fayad, S.G. Worthley, G. Helft, W.F. Chaplin, et al., Effects of aggressive versus conventional lipid-lowering therapy by simvastatin on human atherosclerotic lesions: a prospective, randomized, double-blind trial with highresolution magnetic resonance imaging, J. Am. Coll. Cardiol. 46 (2005) 106-112.

[31] J.A. Lima, M.Y. Desai, H. Steen, W.P. Warren, S. Gautam, S. Lai, Statin-induced cholesterol lowering and plaque regression after 6 months of magnetic resonance imaging-monitored therapy, Circulation 110 (2004) 2336-2341.

[32] J.M. Lee, F. Wiesmann, C. Shirodaria, P. Leeson, S.E. Petersen, J.M. Francis, et al., Early changes in arterial structure and function following statin initiation: quantification by magnetic resonance imaging, Atherosclerosis 197 (2008) 951-958.

[33] J.R. Crouse 3rd, J.S. Raichlen, W.A. Riley, G.W. Evans, M.K. Palmer, D.H. O'Leary, et al., Effect of rosuvastatin on progression of carotid intima-media thickness in low-risk individuals with subclinical atherosclerosis: the METEOR Trial, JAMA 297 (2007) 1344-1353.

[34] X.Q. Zhao, L. Dong, T. Hatsukami, B.A. Phan, B. Chu, A. Moore, et al., MR imaging of carotid plaque composition during lipid-lowering therapy a prospective assessment of effect and time course, J. Am. Coll. Cardiol. Img. 4 (2011) 977-986.

[35] H.R. Underhill, C. Yuan, X.Q. Zhao, L.W. Kraiss, D.L. Parker, T. Saam, et al., Effect of rosuvastatin therapy on carotid plaque morphology and composition in moderately hypercholesterolemic patients: a high-resolution magnetic resonance imaging trial, Am. Heart J. 155 (2008) 584 e1-8.

[36] R. Nakazato, H. Gransar, D.S. Berman, V.Y. Cheng, F.Y. Lin, S. Achenbach, et al., Statins use and coronary artery plaque composition: results from the International Multicenter CONFIRM Registry, Atherosclerosis 225 (2012) 148-153.

[37] L. Pletsch-Borba, M. Selwaness, A. van der Lugt, A. Hofman, O.H. Franco, M.W. Vernooij, Change in carotid plaque components: a 4-year follow-up study with serial MR imaging, JACC Cardiovasc. Imaging 11 (2 Pt 1) (2018) 184-192, https:// doi.org/10.1016/j.jcmg.2016.12.026 [Epub 2017 Apr 12].

[38] D. Proudfoot, J.N. Skepper, L. Hegyi, M.R. Bennett, C.M. Shanahan, P.L. Weissberg, Apoptosis regulates human vascular calcification in vitro: evidence for initiation of vascular calcification by apoptotic bodies, Circ. Res. 87 (2000) 1055-1062.
[39] Y.-W. Wu, H.-L. Kao, C.-L. Huang, M.-F. Chen, L.-Y. Lin, Y.-C. Wang, et al., The effects of 3-month atorvastatin therapy on arterial inflammation, calcification, abdominal adipose tissue and circulating biomarkers, Eur. J. Nucl. Med. Mol. Imaging 39 (2012) 399-407.

[40] A. Tawakol, Z.A. Fayad, R. Mogg, A. Alon, M.T. Klimas, H. Dansky, et al., Intensification of statin therapy results in a rapid reduction in atherosclerotic inflammation, J. Am. Coll. Cardiol. 62 (2013) 909-917.

[41] D. Bos, M.L. Portegies, A. van der Lugt, M.J. Bos, P.J. Koudstaal, A. Hofman, et al., Intracranial carotid artery atherosclerosis and the risk of stroke in whites: the Rotterdam Study, JAMA Neurol. 71 (2014) 405-411.

[42] R. Puri, P. Libby, S.E. Nissen, K. Wolski, C.M. Ballantyne, P.J. Barter, et al., Long-term effects of maximally intensive statin therapy on changes in coronary atheroma composition: insights from SATURN, Eur. Heart J. Cardiovasc. Imaging 15 (2014) 380-388.

[43] A. Keraliya, R. Blankstein, Regression of coronary atherosclerosis with medical therapy, N. Engl. J. Med. 376 (2017) 1370.

[44] A. Pender, D.M. Lloyd-Jones, N.J. Stone, P. Greenland, Refining statin prescribing in lower-risk individuals: informing risk/benefit decisions, J. Am. Coll. Cardiol. 68 (2016) 1690-1697.

[45] S.E. Nissen, S.J. Nicholls, I. Sipahi, P. Libby, J.S. Raichlen, C.M. Ballantyne, et al., Effect of very high-intensity statin therapy on regression of coronary atherosclerosis: the ASTEROID trial, JAMA 295 (2006) 1556-1565.

[46] S.E. Nissen, Effect of intensive lipid lowering on progression of coronary atherosclerosis: evidence for an early benefit from the Reversal of Atherosclerosis with Aggressive Lipid Lowering (REVERSAL) trial, Am. J. Cardiol. 96 (2005) 61F-68F.

[47] S.F. Nielsen, B.G. Nordestgaard, Negative statin-related news stories decrease statin persistence and increase myocardial infarction and cardiovascular mortality: a nationwide prospective cohort study, Eur. Heart J. 37 (2016) 908-916.

[48] A. Irkle, A.T. Vesey, D.Y. Lewis, J.N. Skepper, J.L. Bird, M.R. Dweck, et al., Identifying active vascular microcalcification by (18)F-sodium fluoride positron emission tomography, Nat. Commun. 6 (2015) 7495. 